\title{
ANTI-PEST PROTECTION OF TWO-YEAR OLD ALFALFA GROWN FOR SEEDS
}

Tyshchenko A.V. ${ }^{1}$, Tyshchenko O.D. ${ }^{1}$, Kuts G.M. ${ }^{1}$, Piliarska O.O. ${ }^{1}$, Galchenko N.M. ${ }^{2}$

${ }^{1}$ Institute of Irrigated Agriculture of NAAS, Ukraine

${ }^{2}$ Askania State Agricultural Experimental Station of the Institute of Irrigated Agriculture of NAAS, Ukraine

Purpose. To evaluate the effectiveness of different insecticides against pests on two-year old alfalfa grown for seeds. The highest seed yield was achieved after treatment 1 with an insecticide containing active ingredients chlorpyrifos $500 \mathrm{~g} / \mathrm{L}$ and cypermethrin $50 \mathrm{~g} / \mathrm{L}$ applied at a dose of $1.00 \mathrm{~L} / \mathrm{ha}$ and treatment 2 with insecticides containing active ingredient chlorantraniliprole $200 \mathrm{~g} / \mathrm{L}$ or lambda-cyhalothrin $50 \mathrm{~g} / \mathrm{L}$ applied at a dose of 0.17 and $0.15 \mathrm{~L} / \mathrm{ha}$, respectively.

\section{Key words: alfalfa, seeds, insecticides, irrigation, rainfed conditions.}

Introduction. The primary objective of agriculture is the cultivation of crops, production of food for human and domestic animals. With the increase of the world's population and in order to satisfy its needs, it is necessary to raise the plant performance. Unfortunately, there are pests reducing crop yields intended for human consumption $[1,2,3]$. It is believed that the world produces a sufficient amount of agricultural products that meet the population's needs, but the pestinduced damage to crops leads to severe economic consequences [4]. For example, the presence and number of harmful insects on alfalfa is a factor determining its seed productivity. Depending on various conditions prevailing in each case in the grass canopy, seed yield losses can reach 30-50\% and, in some cases, even more. However, the yield can be significantly increased via timely prevention of losses caused by diseases, pests and weeds $[5,6,7]$. The total insect-induced losses in the yield have increased, and are largely due to changes in farming methods and cultivation technologies.

Effective pest control is very important to minimize damage and, consequently, economic losses, especially in recent years with an increase in the number of warm winters, which are favorable for pest overwintering and development [8]. Different agricultural techniques, sometimes even with growing resistant varieties, do not always ensure desired plant protection, and thus additional control measures, like synthetic chemicals, are needed [9].

However, successful chemical control requires extensive knowledge about the insecticide effects not only on pest (especially vectors) mortality and egg laying, but also on their feeding behavior.

Literature review. Phytosanitary condition of agrocenoses, despite the measures taken, does not improve due to zero or minimal tillage, non-adherence to crop rotations, unbalanced application of mineral fertilizers, insufficient and improper use of pesticides, non-compliance with cultivation technologies [10, 11]. Abate T. and Ampofo J.K.O. claim that there is considerable evidence that pest populations and their numbers are significantly more numerous in monocrops than in crop rotations, while growing two or more crops in the same field simultaneously increases the number of entomophages and usually retains the pest numbers at low levels [12]. Agrotechnological techniques, including site selection, crop rotation, selection of a variety (hybrid) and seeds, timing and method of sowing, can to some extent reduce the numbers of some insects - pests. For example, in a publication [13], researchers reported a decrease in the number of aphids on earlysown wheat. Aheer G.M. et al. [14] showed that sowing time affected the populations of aphids and other pests of legumes. In addition, the number of pests can be regulated by interrow width, plant density and weed control [15]. Forbes V.E. et al. noted that the use of straw and mustard mulch

(C) A.V. Tyshchenko, O.D. Tyshchenko, G.M. Kuts, O.O. Piliarska, N.M. Galchenko. 2021.

ISSN 1026-9959. Селекція і насінництво. 2021. Випуск 119 
reduced the populations and the numbers of insects - bean pests by $75 \%$ [16]. Moreover, it was shown that sloping plots and forest belts reduced wind speed, complicated aphid migrations and, accordingly, impacted its spread, decreasing the affection of plants by viral diseases [17]. Control of vector insects is a serious challenge, so the choice of the most effective insecticides to control them in different environments is always important $[18,19]$.

Insects are a huge group of living organisms, but not many of them are harmful to crops. Insecticides used in crop production are selective and, as a rule, destroy all entomofauna [20]. Alfalfa is often referred to as the beginning of the food chain because it supports not only domestic animals and humans, but also many species of wild animals and birds (more than 700) and more than 1,000 species of arthropods, which is very important for the Earth's ecosystem [21].

Alfalfa grown for seeds is severely damaged by pests, both polyphagous and specialized. The cultivation duration and growing period, its high fodder value and hospitable conditions for pest overwintering favor the settlement of pests and increase in their numbers [22]. Holoborodko SP et al.'s eco-faunal conducted studies in the Left-Bank Lower Dnipro Region of the Southern Steppe of Ukraine, in which they determined and systematized the species composition of the biocenosis of seed alfalfa, with an economic assessment. Complex damage to all organs of seed alfalfa is caused by 157 pest species [23]. Alfalfa is damaged at different stages of plant development - from seed germination to ripening. Pests inflict the most significant damage to seed fields, damaging generative organs and seeds. The pest species composition and numbers on seed alfalfa are influenced by weather during the growing period and winter. Therefore, the absence of protection measures or untimely taken measures dramatically reduces the seed yield and the sowing qualities of seeds [24].

It should be noted that pests, which severely damage two-year old alfalfa grass canopy grown for seeds, are alfalfa weevil (imagoes and grubs), alfalfa plant bug, alfalfa seed weevil, alfalfa seed chalcid, alfalfa aphid, and, in some years, beet webworm. The species composition of insects - pests in alfalfa canopy, their numbers and harmfulness change markedly both zonally and during the alfalfa life in one field as well as during the growing period [25]. However, despite the extensive knowledge on the species composition of entomofauna on seed alfalfa, thorough published information on the pest harmfulness and ecology as well as on integrated protection of alfalfa fields against pest communities and individual species is extremely scarce.

Purpose. To evaluate the effectiveness of different insecticides against pests on two-year old alfalfa grown for seeds.

Materials and methods. The study was conducted in the experimental field of the Institute of Irrigated Agriculture of NAAS in 2017-2019. As to soil and climate, the study site is located in the steppe (Inhulets irrigated massif).

The filed experiments were laid out in split plots. The main plots (factor A) were for water supply conditions (no irrigation vs. irrigation); sub-plots (factors B and C) were for application of insecticides (Table 1). Seeds were sown in wide rows with interrows of $70 \mathrm{~cm}$. The sowing area was $60 \mathrm{~m}^{2}$; the record area was $50 \mathrm{~m}^{2}$; the experiments were carried out in three replications. Alfalfa variety Elehiia was taken. The grass canopy was two years old (sown in spring). Seeds were harvested with the first mowing.

The composition of harmful insect species was determined by surveys using an entomological sweep-net (10 sweeps); their numbers and ratios of different stages were associated with the phases of plant development and weather conditions (temperature, air humidity and precipitation) [26]. The efficiency of timing and numbers of chemical treatments was evaluated by S.O. Tribel's method [27] with due account for the economic thresholds of harmfulness [28].

Insecticides were applied as follows: treatment 1 was at the budding onset; treatment 2 was prior to anthesis with a mounted sprayer ON-600 with operating fluid consumption of $250 \mathrm{~L} / \mathrm{ha}$.

Experimental data were statistically processed in AgroSTAT, XLSTAT and Statistica (v. 13). 
Experiment design

Table 1

\begin{tabular}{|c|c|c|c|}
\hline \multirow{2}{*}{ 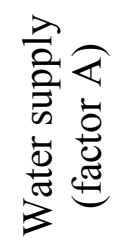 } & \multicolumn{2}{|r|}{ Application of insecticide } & \multirow{2}{*}{ 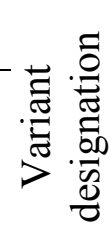 } \\
\hline & $\begin{array}{l}\text { Treatment } 1 \\
\text { (factor B) }\end{array}$ & Treatment 2 (factor $\mathrm{C}$ ) & \\
\hline \multirow{5}{*}{ 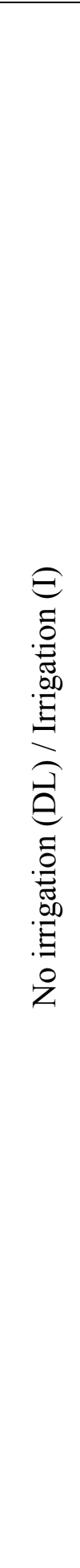 } & 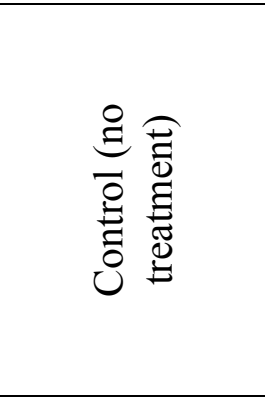 & $\begin{array}{l}\text { Control (no treatment) } \\
\text { Dimethoate } 400 \mathrm{~g} / \mathrm{L}+\text { lambda-cyhalothrin } 50 \mathrm{~g} / \mathrm{L} ; 1.00 \mathrm{~L} / \mathrm{ha}+0.15 \\
\mathrm{~L} / \text { ha } \\
\text { Imidacloprid } 200 \mathrm{~g} / \mathrm{L}+\text { lambda-cyhalothrin } 50 \mathrm{~g} / \mathrm{L} ; 0.20 \mathrm{~L} / \mathrm{ha}+0.15 \\
\mathrm{~L} / \mathrm{ha} \\
\text { Chlorpyrifos } 500 \mathrm{~g} / \mathrm{L}+\text { cypermethrin } 50 \mathrm{~g} / \mathrm{L} ; 1.00 \mathrm{~L} / \mathrm{ha} \\
\text { Chlorantraniliprole } 200 \mathrm{~g} / \mathrm{L}+\text { lambda-cyhalothrin } 50 \mathrm{~g} / \mathrm{L} ; 0.17 \mathrm{~L} / \mathrm{ha} \\
+0.15 \mathrm{~L} / \mathrm{ha}\end{array}$ & $\begin{array}{l}1-3 \\
1-4 \\
1-5\end{array}$ \\
\hline & 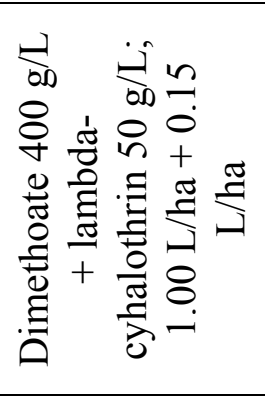 & $\begin{array}{l}\text { Control (no treatment) } \\
\text { Dimethoate } 400 \mathrm{~g} / \mathrm{L}+\text { lambda-cyhalothrin } 50 \mathrm{~g} / \mathrm{L} ; 1.00 \mathrm{~L} / \mathrm{ha}+0.15 \\
\mathrm{~L} / \text { ha } \\
\text { Imidacloprid } 200 \mathrm{~g} / \mathrm{L}+\text { lambda-cyhalothrin } 50 \mathrm{~g} / \mathrm{L} ; 0.20 \mathrm{~L} / \mathrm{ha}+0.15 \\
\mathrm{~L} / \text { ha } \\
\text { Chlorpyrifos } 500 \mathrm{~g} / \mathrm{L}+\text { cypermethrin } 50 \mathrm{~g} / \mathrm{L} ; 1.00 \mathrm{~L} / \mathrm{ha} \\
\text { Chlorantraniliprole } 200 \mathrm{~g} / \mathrm{L}+\text { lambda-cyhalothrin } 50 \mathrm{~g} / \mathrm{L} ; 0.17 \mathrm{~L} / \mathrm{ha} \\
+0.15 \mathrm{~L} / \mathrm{ha}\end{array}$ & $\begin{array}{l}2-3 \\
2-4 \\
2-5\end{array}$ \\
\hline & 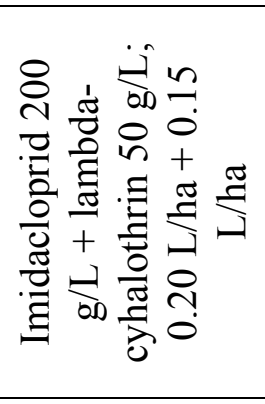 & $\begin{array}{l}\text { Control (no treatment) } \\
\text { Dimethoate } 400 \mathrm{~g} / \mathrm{L}+\text { lambda-cyhalothrin } 50 \mathrm{~g} / \mathrm{L} ; 1.00 \mathrm{~L} / \mathrm{ha}+0.15 \\
\mathrm{~L} / \mathrm{ha} \\
\text { Imidacloprid } 200 \mathrm{~g} / \mathrm{L}+\text { lambda-cyhalothrin } 50 \mathrm{~g} / \mathrm{L} ; 0.20 \mathrm{~L} / \mathrm{ha}+0.15 \\
\mathrm{~L} / \mathrm{ha} \\
\text { Chlorpyrifos } 500 \mathrm{~g} / \mathrm{L}+\text { cypermethrin } 50 \mathrm{~g} / \mathrm{L} ; 1.00 \mathrm{~L} / \mathrm{ha} \\
\text { Chlorantraniliprole } 200 \mathrm{~g} / \mathrm{L}+\text { lambda-cyhalothrin } 50 \mathrm{~g} / \mathrm{L} ; 0.17 \mathrm{~L} / \mathrm{ha} \\
+0.15 \mathrm{~L} / \mathrm{ha}\end{array}$ & $\begin{array}{l}3-3 \\
3-4 \\
3-5\end{array}$ \\
\hline & 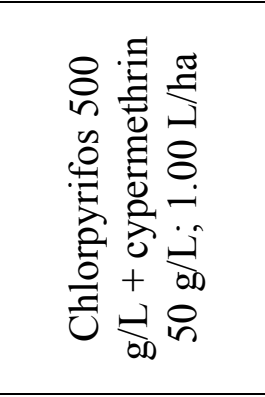 & $\begin{array}{l}\text { Control (no treatment) } \\
\text { Dimethoate } 400 \mathrm{~g} / \mathrm{L}+\text { lambda-cyhalothrin } 50 \mathrm{~g} / \mathrm{L} ; 1.00 \mathrm{~L} / \mathrm{ha}+0.15 \\
\mathrm{~L} / \mathrm{ha} \\
\text { Imidacloprid } 200 \mathrm{~g} / \mathrm{L}+\text { lambda-cyhalothrin } 50 \mathrm{~g} / \mathrm{L} ; 0.20 \mathrm{~L} / \mathrm{ha}+0.15 \\
\mathrm{~L} / \mathrm{ha} \\
\text { Chlorpyrifos } 500 \mathrm{~g} / \mathrm{L}+\text { cypermethrin } 50 \mathrm{~g} / \mathrm{L} ; 1.00 \mathrm{~L} / \mathrm{ha} \\
\text { Chlorantraniliprole } 200 \mathrm{~g} / \mathrm{L}+\text { lambda-cyhalothrin } 50 \mathrm{~g} / \mathrm{L} ; 0.17 \mathrm{~L} / \mathrm{ha} \\
+0.15 \mathrm{~L} / \mathrm{ha}\end{array}$ & $\begin{array}{l}4-3 \\
4-4 \\
4-5\end{array}$ \\
\hline & 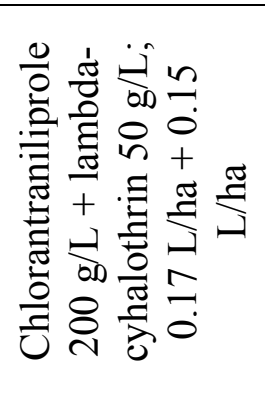 & $\begin{array}{l}\text { Control (no treatment) } \\
\text { Dimethoate } 400 \mathrm{~g} / \mathrm{L}+\text { lambda-cyhalothrin } 50 \mathrm{~g} / \mathrm{L} ; 1.00 \mathrm{~L} / \mathrm{ha}+0.15 \\
\mathrm{~L} / \mathrm{ha} \\
\text { Imidacloprid } 200 \mathrm{~g} / \mathrm{L}+\text { lambda-cyhalothrin } 50 \mathrm{~g} / \mathrm{L} ; 0.20 \mathrm{~L} / \mathrm{ha}+0.15 \\
\mathrm{~L} / \mathrm{ha} \\
\text { Chlorpyrifos } 500 \mathrm{~g} / \mathrm{L}+\text { cypermethrin } 50 \mathrm{~g} / \mathrm{L} ; 1.00 \mathrm{~L} / \mathrm{ha} \\
\text { Chlorantraniliprole } 200 \mathrm{~g} / \mathrm{L}+\text { lambda-cyhalothrin } 50 \mathrm{~g} / \mathrm{L} ; 0.17 \mathrm{~L} / \mathrm{ha} \\
+0.15 \mathrm{~L} / \mathrm{ha}\end{array}$ & $\begin{array}{l}5-3 \\
5-4\end{array}$ \\
\hline
\end{tabular}

Results and discussion. Several pest species were found on alfalfa, differing in peculiarities of their development and damage to plants. When the two-year old alfalfa canopy (budding onset) 
was inspected before insecticide treatment, the average numbers of pests were as follows: alfalfa plant bug -3.0 specimens/10 sweeps, alfalfa aphid -20.0 specimens/10 sweeps, beet webworm 3.0 specimens/10 sweeps, alfalfa weevil (imagoes/grubs) - 1.0/3.0 specimens/10 sweeps, and alfalfa seed weevil - 1.0 specimens/10 sweeps (Table 2). It is known that the effectiveness of different insecticides against mandibulates and sucking pests is not the same, so there is a need to assess the effectiveness of universal and binary mixtures of insecticides against the pest complex.

Treatment 1 reduced the pest numbers in the canopy: alfalfa plant bug - by $70.0-93.3 \%$, alfalfa aphid - by $93.0-97.5 \%$, beet webworm - by $80.0-96.7$, alfalfa weevil (imagoes/grubs) - by 60.0-90.0\%/73.3-93.3\%, and alfalfa seed weevil - by 76.0-94.0\%, depending on the insecticide.

Table 2

Pest numbers before and after the application of insecticides and their effectiveness on twoyear old alfalfa grown for seeds, (2017-2019 average)

\begin{tabular}{|c|c|c|c|c|c|c|c|c|c|c|c|c|c|c|c|c|c|c|}
\hline \multirow{3}{*}{$\stackrel{\vec{E}}{\vec{E}}$} & \multicolumn{12}{|c|}{ Pest numbers, specimens/10 sweeps } & \multicolumn{6}{|c|}{ Reduction in the pest numbers, $\%$} \\
\hline & \multicolumn{2}{|c|}{ 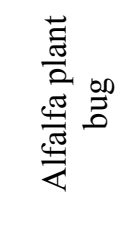 } & \multicolumn{2}{|c|}{ 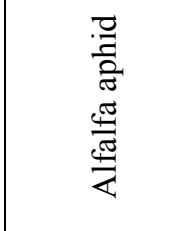 } & \multicolumn{2}{|c|}{$\begin{array}{l}\Xi \\
0 \\
0 \\
0 \\
0 \\
3 \\
0 \\
0 \\
0\end{array}$} & \multicolumn{2}{|c|}{ 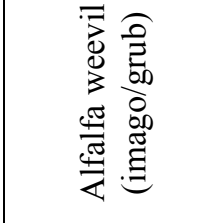 } & \multicolumn{2}{|c|}{ 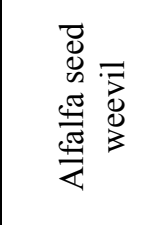 } & \multicolumn{2}{|c|}{ 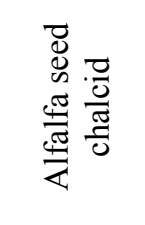 } & \multirow{2}{*}{ 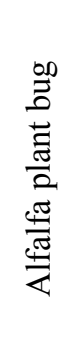 } & \multirow{2}{*}{ 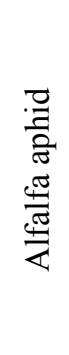 } & \multirow{2}{*}{$\begin{array}{l}E \\
0 \\
0 \\
3 \\
0 \\
0 \\
0 \\
0 \\
0 \\
0\end{array}$} & \multirow{2}{*}{ 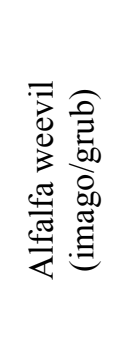 } & \multirow{2}{*}{ 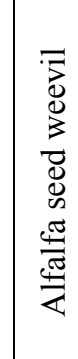 } & \multirow{2}{*}{ 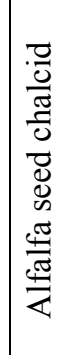 } \\
\hline & $\begin{array}{c}\text { befor } \\
\text { e }\end{array}$ & $\begin{array}{l}\text { af- } \\
\text { ter }\end{array}$ & before & after & $\begin{array}{c}\text { befor } \\
\text { e }\end{array}$ & after & before & after & $\begin{array}{c}\text { befor } \\
\text { e }\end{array}$ & after & $\begin{array}{c}\text { befo } \\
\text { re }\end{array}$ & after & & & & & & \\
\hline \multicolumn{19}{|c|}{ Treatment 1} \\
\hline 1 & \multirow{5}{*}{3.0} & 4.0 & \multirow{5}{*}{20.0} & 25.0 & \multirow{5}{*}{3.0} & 4.0 & \multirow{5}{*}{$1.0 / 3.0$} & $2.0 / 5.0$ & \multirow{5}{*}{1.0} & 2.0 & \multirow{5}{*}{0.0} & 0.0 & - & - & - & - & - & - \\
\hline 2 & & 0.9 & & 0.5 & & 0.6 & & $0.4 / 0.8$ & & 0.3 & & 0.0 & 70.0 & 97.5 & 80.0 & \begin{tabular}{|l|}
$60.0 / 73.3$ \\
\end{tabular} & 70.0 & - \\
\hline 3 & & 0.5 & & 1.4 & & 0.4 & & $0.2 / 0.4$ & & 0.2 & & 0.0 & 83.3 & 93.0 & 86.7 & $80.0 / 86.7$ & 80.0 & - \\
\hline 4 & & 0.2 & & 0.8 & & 0.1 & & $0.1 / 0.2$ & & 0.1 & & 0.0 & 93.3 & 96.0 & 96.7 & \begin{tabular}{|l|}
$90.0 / 93.3$ \\
\end{tabular} & 90.0 & - \\
\hline 5 & & 0.3 & & 1.0 & & 0.3 & & $0.2 / 0.3$ & & 0.2 & & 0.0 & 90.0 & 95.0 & 90.0 & $80.0 / 90.0$ & 80.0 & - \\
\hline & & & & & & & & Tre & tment & & & & & & & & & \\
\hline $1-1$ & & 2.1 & & 10.0 & & 2.0 & & $0.0 / 0.0$ & & 3.8 & & 4.7 & - & - & - & - & - & - \\
\hline $1-2$ & & 0.8 & & 0.2 & & 0.8 & & $0.0 / 0.0$ & & 1.0 & & 0.9 & 60.0 & 98.8 & 80.0 & - & 66.7 & 77.5 \\
\hline $1-3$ & 2.0 & 0.6 & 17.0 & 0.7 & 4.0 & 0.1 & $0.3 / 0.0$ & $0.0 / 0.0$ & 3.0 & 0.7 & 4.0 & 0.6 & 70.0 & 95.9 & 97.5 & - & 76.7 & 85.0 \\
\hline $1-4$ & & 0.3 & & 0.3 & & 0.1 & & $0.0 / 0.0$ & & 0.3 & & 0.3 & 85.0 & 98.2 & 97.5 & - & \begin{tabular}{l|l|}
90.0 \\
\end{tabular} & 92.5 \\
\hline $1-5$ & & 0.4 & & 0.5 & & 0.1 & & $0.0 / 0.0$ & & 0.5 & & 0.4 & 80.0 & 97.1 & 97.5 & - & \begin{tabular}{|l|l|}
83.3 \\
\end{tabular} & 90.0 \\
\hline $2-1$ & & 1.4 & & 2.1 & & 0.2 & & $0.0 / 0.0$ & & 2.9 & & 3.7 & - & - & - & - & - & - \\
\hline $2-2$ & & 0.5 & & 0.0 & & 0.0 & & $0.0 / 0.0$ & & 0.5 & & 0.7 & 58.3 & 100.0 & 100.0 & - & \begin{tabular}{|l|}
70.6 \\
\end{tabular} & 80.0 \\
\hline $2-3$ & 1.2 & 0.3 & 3.3 & 0.1 & 0.6 & 0.0 & $0.0 / 0.0$ & $0.0 / 0.0$ & 1.7 & 0.4 & 3.5 & 0.4 & 75.0 & 97.0 & 100.0 & - & \begin{tabular}{|c|}
76.5 \\
\end{tabular} & 88.6 \\
\hline $2-4$ & & 0.1 & & 0.0 & & 0.0 & & $0.0 / 0.0$ & & 0.2 & & 0.2 & 91.7 & 100.0 & 100.0 & - & 88.2 & 94.3 \\
\hline $2-5$ & & 0.2 & & 0.0 & & 0.0 & & $0.0 / 0.0$ & & 0.3 & & 0.3 & 83.3 & 100.0 & 100.0 & - & \begin{tabular}{|l|}
82.4 \\
\end{tabular} & 91.4 \\
\hline $3-1$ & & 1.3 & & 3.9 & & 0.1 & & $0.0 / 0.0$ & & 2.0 & & 3.5 & - & 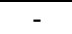 & 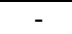 & - & - & - \\
\hline $3-2$ & & 0.4 & & 0.0 & & 0.0 & & $0.0 / 0.0$ & & 0.4 & & 0.6 & 60.0 & 100.0 & 100.0 & - & \begin{tabular}{|l|}
71.4 \\
\end{tabular} & 80.6 \\
\hline $3-3$ & 1.0 & 0.2 & 5.4 & 0.2 & 0.4 & 0.0 & $0.0 / 0.0$ & $0.0 / 0.0$ & 1.4 & 0.3 & 3.1 & 0.3 & 80.0 & 96.3 & 100.0 & - & 78.6 & 90.3 \\
\hline $3-4$ & & 0.1 & & 0.1 & & 0.0 & & $0.0 / 0.0$ & & 0.1 & & 0.2 & 90.0 & \begin{tabular}{|l}
98.1 \\
\end{tabular} & 100.0 & - & \begin{tabular}{|l|}
92.9 \\
\end{tabular} & 93.5 \\
\hline $3-5$ & & 0.2 & & 0.2 & & 0. & & $0.0 / 0.0$ & & 0.2 & & 0. & 80.0 & 96.3 & 100.0 & - & 85.7 & 90.3 \\
\hline $4-1$ & & 1.0 & & 3.4 & & 0.0 & & $0.0 / 0.0$ & & 1.6 & & 3. & - & - & - & - & - & - \\
\hline $4-2$ & & 0.3 & & 0.0 & & 0.0 & & $0.0 / 0.0$ & & 0.3 & & 0.5 & 62.5 & 100.0 & 100.0 & - & 70.0 & 81.5 \\
\hline $4-3$ & 0.8 & 0.1 & 3.6 & 0.0 & 0.1 & 0.0 & $0.0 / 0.0$ & $0.0 / 0.0$ & 1.0 & 0.2 & 2.7 & 0.3 & 87.5 & 100.0 & 100.0 & - & \begin{tabular}{|l|}
80.0 \\
\end{tabular} & 88.9 \\
\hline $4-4$ & & 0.1 & & 0.0 & & 0.0 & & $0.0 / 0.0$ & & 0.1 & & 0.2 & 87.5 & 100.0 & 100.0 & - & \begin{tabular}{|l|}
90.0 \\
\end{tabular} & 92.6 \\
\hline $4-5$ & & 0.1 & & 0.0 & & 0.0 & & $0.0 / 0.0$ & & 0.1 & & 0.2 & 87.5 & 100.0 & 100.0 & - & 90.0 & 92.6 \\
\hline $5-1$ & & 1.2 & & 3.5 & & 0.0 & & $0.0 / 0.0$ & & 1.7 & & 3.3 & - & - & - & - & - & - \\
\hline $5-2$ & & 0.4 & & 0.0 & & 0.0 & & $0.0 / 0.0$ & & 0.4 & & 0.6 & 60.0 & 100.0 & 100.0 & - & 69.2 & 80.0 \\
\hline $5-3$ & 1.0 & 0.2 & 4.0 & 0.2 & 0.3 & 0.0 & $0.0 / 0.0$ & $0.0 / 0.0$ & 1.3 & 0.3 & 3.0 & 0.4 & 80.0 & 95.0 & 100.0 & - & 76.9 & 86.7 \\
\hline $5-4$ & & 0.1 & & 0.0 & & 0.0 & & $0.0 / 0.0$ & & 0.1 & & 0.1 & 90.0 & 100.0 & 100.0 & - & 92.3 & 96.7 \\
\hline $5-5$ & & 0.1 & & 0.1 & & 0.0 & & $0.0 / 0.0$ & & 0.2 & & 0.3 & 90.0 & 97.5 & 100.0 & - & \begin{tabular}{|l|l|}
84.6 \\
\end{tabular} & 90.0 \\
\hline
\end{tabular}

Insecticide containing active substances chlorpyrifos $500 \mathrm{~g} / \mathrm{L}$ and cypermethrin $50 \mathrm{~g} / \mathrm{L}$ applied at a dose of $1.00 \mathrm{~L} /$ ha was the most effective for pest control (except for alfalfa aphids). It reduced the numbers of alfalfa plant bug - by $93.3 \%$, alfalfa aphid - by $96.0 \%$, beet webworm - by $96.7 \%$, alfalfa weevil (imago/grub) - by $90.0 / 93.3 \%$ and alfalfa seed weevil - by $90.0 \%$, and before treatment 2 (prior to anthesis), the pest numbers were the lowest compared to the other variants. 
Treatment 2 helped to reduce the pest numbers in the canopy. Insecticide containing active substances chlorpyrifos $500 \mathrm{~g} / \mathrm{L}$ and cypermethrin $50 \mathrm{~g} / \mathrm{L}$ applied at a dose of $1.00 \mathrm{~L} / \mathrm{ha}$ was the most effective. It reduced the numbers of alfalfa plant bug - by $85.0-91.7 \%$, alfalfa aphid - by $85.0-91.7 \%$, beet webworm - by $97.5 \%$, alfalfa seed weevil - by $88.2-92.3 \%$ and alfalfa seed chalcid - by $92.5-96.7 \%$, depending on the insecticide in treatment 1 (1-4; $2-4 ; 3-4 ; 4-4 ; 5-4)$.

Under rainfed conditions and with application of this insecticide, alfalfa seed weevil-inflicted damage to seeds was $0.22-0.28$ seeds/pod and alfalfa seed chalcidinflicted damage was $0.08-0.10$ seeds/pod, depending on the insecticide in treatment 1 . When irrigated, these indicators were: 0.19 0.24 seeds/pod and 0.06-0.08 seeds/pod, respectively (Table 3). The low pest numbers or their absences usually positively affect the development of generative organs and, accordingly, the seed productivity of plants. However, the fumigation action of this insecticide had a negative effect on the numbers of pollinating insects, interfering with the formation of pods and seeds in generative organs and further affecting the plant performance. Thus, under rainfed conditions, 6.7-7.2 pods/raceme and 2.44-2.69 seeds/pod were formed, and under irrigation, there were 7.6-8.0 pods/raceme and 3.47-4.00 seeds/pod, depending on the insecticide in treatment 1 . At the same time, the under rainfed conditions, the seed yield was $330.53-390.61 \mathrm{~kg} / \mathrm{ha}$, exceeding the control by $10.54-70.62 \mathrm{~kg} / \mathrm{ha}$. When irrigated, the seed yield was $437.63-535.96 \mathrm{~kg} / \mathrm{ha}$, exceeding the control by $13.03-111.36 \mathrm{~kg} / \mathrm{ha}$.

Table 3

Yield structure, seed yield and preserved alfalfa yield, depending on the insecticide application and water supply (2017-2019 average)

\begin{tabular}{|c|c|c|c|c|c|c|c|c|c|c|}
\hline \multirow{3}{*}{ Variant } & \multirow{3}{*}{ 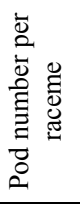 } & \multicolumn{6}{|c|}{ Seed number per pod } & \multirow{3}{*}{ 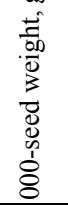 } & \multirow{3}{*}{ 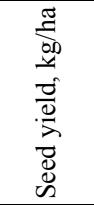 } & \multirow{3}{*}{ 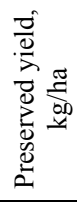 } \\
\hline & & \multirow{2}{*}{ 㶾 } & \multicolumn{2}{|c|}{ Intact } & \multicolumn{3}{|c|}{ Damaged by } & & & \\
\hline & & & Fullvalue & Shrivelled & Total & $\begin{array}{c}\text { Alfalfa seed } \\
\text { weevil }\end{array}$ & $\begin{array}{c}\text { Alfalfa seed } \\
\text { chalcid }\end{array}$ & & & \\
\hline DL 1-1 & 6.6 & 2.66 & 1.87 & 0.79 & 0.59 & 0.46 & 0.13 & 1.92 & 319.99 & - \\
\hline DL 1-2 & 6.7 & 2.70 & 2.06 & 0.64 & 0.47 & 0.36 & 0.11 & 1.93 & 357.73 & 37.74 \\
\hline DL 1-3 & 6.8 & 2.70 & 2.11 & 0.59 & 0.44 & 0.33 & 0.11 & 1.93 & 366.34 & 46.35 \\
\hline DL 1-4 & 6.7 & 2.44 & 1.93 & 0.52 & 0.38 & 0.28 & 0.10 & 1.94 & 330.53 & 10.54 \\
\hline DL 1-5 & 6.9 & 2.69 & 2.19 & 0.50 & 0.37 & 0.28 & 0.09 & 1.94 & 382.79 & 62.80 \\
\hline Mean & 6.73 & 2.64 & 2.03 & 0.61 & 0.45 & 0.34 & 0.11 & 1.93 & 351.48 & - \\
\hline DL 2-1 & 6.9 & 2.76 & 2.06 & 0.71 & 0.52 & 0.41 & 0.12 & 1.93 & 352.16 & 32.17 \\
\hline DL 2-2 & 7.0 & 2.84 & 2.26 & 0.57 & 0.42 & 0.32 & 0.10 & 1.94 & 393.72 & 73.73 \\
\hline DL 2-3 & 7.0 & 2.85 & 2.32 & 0.53 & 0.39 & 0.29 & 0.10 & 1.94 & 403.17 & 83.18 \\
\hline DL 2-4 & 6.9 & 2.58 & 2.12 & 0.46 & 0.34 & 0.25 & 0.09 & 1.95 & 363.72 & 43.73 \\
\hline DL 2-5 & 7.1 & 2.85 & 2.41 & 0.44 & 0.33 & 0.25 & 0.08 & 1.95 & 421.28 & 101.29 \\
\hline Mean & 6.97 & 2.78 & 2.23 & 0.54 & 0.40 & 0.30 & 0.10 & 1.94 & 386.81 & - \\
\hline DL 3-1 & 7.0 & 2.79 & 2.13 & 0.66 & 0.49 & 0.38 & 0.11 & 1.95 & 365.40 & 45.41 \\
\hline DL 3-2 & 7.1 & 2.89 & 2.35 & 0.54 & 0.40 & 0.30 & 0.09 & 1.96 & 408.55 & 88.56 \\
\hline DL 3-3 & 7.2 & 2.90 & 2.40 & 0.50 & 0.37 & 0.28 & 0.10 & 1.96 & 418.35 & 98.36 \\
\hline DL 3-4 & 7.0 & 2.63 & 2.20 & 0.43 & 0.32 & 0.24 & 0.08 & 1.97 & 377.39 & 57.40 \\
\hline DL 3-5 & 7.3 & 2.91 & 2.50 & 0.42 & 0.31 & 0.23 & 0.08 & 1.97 & 437.17 & 117.18 \\
\hline Mean & 7.12 & 2.83 & 2.32 & 0.51 & 0.38 & 0.28 & 0.09 & 1.96 & 401.37 & - \\
\hline DL 4-1 & 7.2 & 2.84 & 2.20 & 0.64 & 0.47 & 0.36 & 0.10 & 1.96 & 378.20 & 58.21 \\
\hline DL 4-2 & 7.3 & 2.94 & 2.43 & 0.51 & 0.38 & 0.29 & 0.09 & 1.96 & 422.86 & 102.87 \\
\hline DL 4-3 & 7.3 & 2.96 & 2.49 & 0.47 & 0.35 & 0.26 & 0.09 & 1.97 & 433.01 & 113.02 \\
\hline DL 4-4 & 7.2 & 2.69 & 2.28 & 0.41 & 0.30 & 0.22 & 0.08 & 1.98 & 390.61 & 70.62 \\
\hline DL 4-5 & 7.4 & 2.98 & 2.59 & 0.40 & 0.29 & 0.22 & 0.07 & 1.98 & 452.48 & 132.49 \\
\hline Mean & 7.26 & 2.88 & 2.40 & 0.49 & 0.36 & 0.27 & 0.09 & 1.97 & 415.43 & - \\
\hline DL 5-1 & 7.0 & 2.76 & 2.09 & 0.67 & 0.49 & 0.38 & 0.11 & 1.94 & 358.56 & 38.57 \\
\hline DL 5-2 & 7.0 & 2.85 & 2.31 & 0.54 & 0.40 & 0.30 & 0.10 & 1.95 & 400.87 & 80.88 \\
\hline DL 5-3 & 7.1 & 2.86 & 2.36 & 0.50 & 0.37 & 0.27 & 0.10 & 1.95 & 410.50 & 90.51 \\
\hline DL 5-4 & 7.0 & 2.59 & 2.16 & 0.43 & 0.32 & 0.24 & 0.08 & 1.96 & 370.33 & 50.34 \\
\hline DL 5-5 & 7.2 & 2.87 & 2.45 & 0.42 & 0.31 & 0.23 & 0.08 & 1.96 & 428.94 & 108.95 \\
\hline Mean & 7.05 & 2.79 & 2.27 & 0.51 & 0.38 & 0.29 & 0.09 & 1.95 & 393.84 & - \\
\hline Mean & 7.03 & 2.78 & 2.25 & 0.53 & 0.39 & 0.30 & 0.10 & 1.95 & 389.78 & - \\
\hline I 1-1 & 7.7 & 3.68 & 2.80 & 0.59 & 0.47 & 0.37 & 0.10 & 2.07 & 424.60 & - \\
\hline I 1-2 & 7.9 & 3.89 & 3.16 & 0.49 & 0.39 & 0.30 & 0.09 & 2.07 & 478.96 & 54.36 \\
\hline I 1-3 & 8.0 & 3.93 & 3.26 & 0.45 & 0.36 & 0.28 & 0.08 & 2.08 & 493.26 & 68.66 \\
\hline I 1-4 & 7.6 & 3.47 & 2.89 & 0.39 & 0.31 & 0.24 & 0.08 & 2.08 & 437.63 & 13.03 \\
\hline I 1-5 & 8.3 & 3.99 & 3.42 & 0.38 & 0.30 & 0.23 & 0.07 & 2.08 & 518.73 & 94.13 \\
\hline Mean & 7.89 & 3.79 & 3.10 & 0.46 & 0.37 & 0.28 & 0.08 & 2.07 & 470.64 & - \\
\hline
\end{tabular}




\begin{tabular}{|c|c|c|c|c|c|c|c|c|c|c|}
\hline I 2-1 & 7.9 & 3.93 & 3.13 & 0.54 & 0.43 & 0.33 & 0.09 & 2.08 & 474.43 & 49.83 \\
\hline I 2-2 & 8.1 & 4.19 & 3.53 & 0.44 & 0.35 & 0.27 & 0.08 & 2.08 & 535.20 & 110.6 \\
\hline I 2-3 & 8.3 & 4.25 & 3.64 & 0.41 & 0.33 & 0.25 & 0.08 & 2.08 & 551.20 & 126.6 \\
\hline I 2-4 & 7.8 & 3.76 & 3.22 & 0.36 & 0.28 & 0.21 & 0.07 & 2.09 & 489.02 & 64.42 \\
\hline I $2-5$ & 8.4 & 4.34 & 3.82 & 0.35 & 0.28 & 0.21 & 0.06 & 2.08 & 579.66 & 155.06 \\
\hline Mean & 8.08 & 4.09 & 3.47 & 0.42 & 0.33 & 0.26 & 0.08 & 2.08 & 525.90 & - \\
\hline I 3-1 & 8.1 & 4.02 & 3.28 & 0.49 & 0.39 & 0.31 & 0.09 & 2.08 & 497.31 & 72.71 \\
\hline I 3-2 & 8.3 & 4.30 & 3.70 & 0.41 & 0.32 & 0.25 & 0.07 & 2.09 & 561.02 & 136.42 \\
\hline I 3-3 & 8.4 & 4.37 & 3.81 & 0.38 & 0.30 & 0.23 & 0.07 & 2.09 & 577.77 & 153.17 \\
\hline I 3-4 & 8.0 & 3.87 & 3.38 & 0.33 & 0.26 & 0.20 & 0.06 & 2.10 & 512.67 & 88.07 \\
\hline I 3-5 & 8.7 & 4.48 & 4.01 & 0.32 & 0.25 & 0.19 & 0.06 & 2.09 & 607.61 & 183.01 \\
\hline Mean & 8.27 & 4.21 & 3.63 & 0.39 & 0.31 & 0.24 & 0.07 & 2.09 & 551.28 & - \\
\hline I 4-1 & 8.2 & 4.14 & 3.43 & 0.48 & 0.38 & 0.30 & 0.08 & 2.09 & 519.86 & 95.26 \\
\hline I 4-2 & 8.4 & 4.45 & 3.87 & 0.39 & 0.31 & 0.24 & 0.07 & 2.09 & 586.46 & 161.86 \\
\hline I 4-3 & 8.5 & 4.52 & 3.98 & 0.36 & 0.29 & 0.22 & 0.07 & 2.10 & 603.97 & 179.37 \\
\hline I 4-4 & 8.0 & 4.00 & 3.53 & 0.32 & 0.25 & 0.19 & 0.06 & 2.10 & 535.96 & 111.36 \\
\hline I 4-5 & 8.8 & 4.64 & 4.19 & 0.31 & 0.24 & 0.19 & 0.06 & 2.10 & 635.16 & 210.56 \\
\hline Mean & 8.38 & 4.35 & 3.80 & 0.37 & 0.30 & 0.23 & 0.07 & 2.10 & 576.28 & - \\
\hline I 5-1 & 8.0 & 3.96 & 3.20 & 0.51 & 0.40 & 0.32 & 0.09 & 2.08 & 485.61 & 61.01 \\
\hline I 5-2 & 8.2 & 4.24 & 3.62 & 0.42 & 0.33 & 0.26 & 0.08 & 2.08 & 547.79 & 123.19 \\
\hline I 5-3 & 8.4 & 4.30 & 3.72 & 0.39 & 0.31 & 0.23 & 0.07 & 2.09 & 564.16 & 139.56 \\
\hline I 5-4 & 7.9 & 3.80 & 3.30 & 0.34 & 0.27 & 0.20 & 0.07 & 2.09 & 500.53 & 75.93 \\
\hline I 5-5 & 8.6 & 4.40 & 3.91 & 0.33 & 0.26 & 0.20 & 0.06 & 2.09 & 593.29 & 168.69 \\
\hline Mean & 8.19 & 4.14 & 3.55 & 0.40 & 0.31 & 0.24 & 0.07 & 2.09 & 538.28 & - \\
\hline Mean & 8.16 & 4.12 & 3.51 & 0.41 & 0.32 & 0.25 & 0.07 & 2.09 & 532.47 & - \\
\hline \multicolumn{11}{|c|}{ Significance of partial differences } \\
\hline A & 0.147 & 5.502 & 4.959 & 0.587 & 0.264 & 0.161 & 0.015 & 0.161 & 61.318 & \\
\hline $\mathrm{B}$ & 0.024 & 0.040 & 0.050 & 0.012 & 0.009 & 0.005 & 0.002 & 0.012 & 1.118 & \\
\hline $\mathrm{C}$ & 0.023 & 0.022 & 0.025 & 0.013 & 0.014 & 0.008 & 0.002 & 0.004 & 0.719 & \\
\hline \multicolumn{11}{|c|}{ Significance of the means (main effects) } \\
\hline A & 0.017 & 0.635 & 0.573 & 0.068 & 0.030 & 0.019 & 0.002 & 0.019 & 7.080 & \\
\hline $\mathrm{B}$ & 0.004 & 0.007 & 0.009 & 0.002 & 0.002 & 0.001 & 0.001 & 0.002 & 0.204 & \\
\hline $\mathrm{C}$ & 0.004 & 0.004 & 0.005 & 0.002 & 0.003 & 0.002 & 0.001 & 0.001 & 0.131 & \\
\hline
\end{tabular}

Note: $\mathrm{RF}=$ rainfed; $\mathrm{I}=$ irrigatred.

The highest seed yield was achieved after treatment 1 with insecticide containing active ingredients chlorpyrifos $500 \mathrm{~g} / \mathrm{L}$ and cypermethrin $50 \mathrm{~g} / \mathrm{L}$ applied at a dose of $1.00 \mathrm{~L} / \mathrm{ha}$ and treatment 2 with insecticide containing active ingredients chlorantraniliprole $200 \mathrm{~g} / \mathrm{L}$ and lambdacyhalothrin $50 \mathrm{~g} / \mathrm{L}$ applied at a dose of $0.17 \mathrm{~L} / \mathrm{ha}$ and $0.15 \mathrm{~L} / \mathrm{ha}$, respectively. With irrigation on this variant, 8.8 pods/raceme and 4.64 seeds/pod were produced, and the seed productivity was 635.16 $\mathrm{kg} / \mathrm{ha}$, which was higher than the control value by $210.56 \mathrm{~kg} / \mathrm{ha}$. Under rainfed conditions, plants formed 7.4 pods $/$ raceme and 2.98 seeds/pod. Here, the seed yield was $452.48 \mathrm{~kg} / \mathrm{ha}$, exceeding the control by $132.49 \mathrm{~kg} / \mathrm{ha}$.

Conclusions. Insecticide containing active substances chlorpyrifos $500 \mathrm{~g} / \mathrm{L}$ and cypermethrin $50 \mathrm{~g} / \mathrm{L}$ applied at a dose of $1.00 \mathrm{~L} / \mathrm{ha}$ was the most effective for pest control (except for alfalfa aphids). However, the fumigation action of this insecticide had a negative effect on the numbers of pollinating insects, interfering with the formation of pods and seeds and further affecting the plant performance, so this insecticide should not be used prior to anthesis (treatment 2). The highest seed yield was achieved after treatment 1 with insecticide containing active ingredients chlorpyrifos 500 $\mathrm{g} / \mathrm{L}$ and cypermethrin $50 \mathrm{~g} / \mathrm{L}$ applied at a dose of $1.00 \mathrm{~L} / \mathrm{ha}$ and treatment 2 with insecticide containing active ingredients chlorantraniliprole $200 \mathrm{~g} / \mathrm{L}$ and lambda-cyhalothrin $50 \mathrm{~g} / \mathrm{L}$ applied at a dose of $0.17 \mathrm{~L} / \mathrm{ha}$ and $0.15 \mathrm{~L} / \mathrm{ha}$, respectively.

\section{Список використаних джерел}

1. Katungi E. et al. Common bean in Eastern and Southern Africa: A Situation and Outlook Analysis. International Centre for Tropical Agriculture, 2009. $61 \mathrm{p}$.

2. Ochilo W.N., Nyamasyo G.H. Pest status of bean stem maggot (Ophiomyia spp.) and black bean aphid (Aphis fabae) in Taita district, Kenya. Tropical and Subtropical Agro Ecosystems. 2011. Vol. 13(1). P. 91-97.

3. Munyasa A.J. Evaluation of drought tolerance mechanisms in mesoamerican dry bean genotypes. University of Nairobi, Nairobi. 2013. 191 p.

4. Спиридонов Ю.Я. и др. Технология возделывания яровой твердой пшеницы с 
применением препаратов Секатор турбо, Баритон, Фалькон, Нагро и других. Аграрный научный журнал. 2017. № 3. C. 30-36. DOI: 10.28983/asj.v0i3.47.

5. Стрижков Н.И. и др. Интегрированная технология защиты посевов полевых культур от болезней, вредителей и сорняков на основе биологических и химических методов. Саратов, 2017. 56 c.

6. Спиридонов Ю.Я. и др. Разработка интегрированной технологии защиты посевов полевых культур от болезней, вредителей и сорняков на основе биологических и химических методов. Аграрный научный журнал. 2017. № 9. С. 37-42.

7. Дудкин И.В. и др. Экологические аспекты формирования систем земледелия и защиты растений. Вестник Курской государственной сельскохозяйственной академии. 2017. № 7. C. 2-7.

8. Peter K.H., Swella G.B., and Mushobozy D.M. Effect of plant populations on the incidence of bean stem maggot (Ophiomyia spp.) in common bean intercropped with maize. Plant Protection Science. 2009. Vol. 45(4). P. 148-155. DOI: 10.17221/19/2009-PPS.

9. Dhungana S.K. et al. Comparative effect of different insecticides on the growth and yield of soybeans. Plant Protect. Sci. 2020. Vol. 56: 206-213. DOI: 10.17221/77/2019-PPS.

10. Худенко М.Н. и др. Продуктивность расторопши пятнистой в зависимости от способов обработки почвы и химических средств защиты в сухой степи Поволжья. Аграрный научный журнал. 2016. № 12. С. 43-49.

11. Спиридонов Ю.Я. и др. Особенности влияния химических средств защиты растений на динамику элементов питания в растениях, их химический состав и условия развития. Аграрный научный журнал. 2018. №10. C 37-40. DOI: 10.28983/asj.v0i10.606.

12. Abate T. and Ampofo J.K.O. Insect pests of beans in Africa: their ecology and management. Annual Review of Entomology. 1996. Vol. 41. P. 45-73. http://dx.doi.org/10.1146/annurev.en.41.010196.000401.

13. Acreman T.M. and Dixon A.F. Developmental patterns in the wheat and resistant to Cereal Aphids. Crop Protect. 1985. Vol. 4. P. 322-328. http://dx.doi.org/10.1016/02612194(85)90034-1.

14. Aheer G.M. et al. Effects of swoing dates on aphids and grain yield in wheat. Journal of Agricultural Research. 1993. Vol. 31. P. 75-79.

15. Nderitu J.H., Kayumbo H.Y. and Mueke J.M. Beanfly infestation on common beans Phaseolus vulgaris in Kenya. Insect Science and Its Application. 1990. Vol. 11. P. 35-41. DOI: https://doi.org/10.1017/S1742758400019810.

16. Forbes V.E. et al. Ecological models in support of regulatory risk assessments of pesticides: developing a strategy for the future. Integrated Environmental Assessment and Management. 2009. Vol. 5. P. 167-172. DOI: 10.1897/ieam_2008-029.1.

17. Stoddard F.L. et al. Integrated pest management in faba bean. Field Crops Research. 2010. Vol. 115. P. 308-318. DOI: 10.1016/j.fcr.2009.07.002.

18. Maluta N.K.P. et al. Foliar spraying of tomato plants with systemic insecticides: effects on feeding behavior, mortality and oviposition of Bemisia tabaci (Hemiptera: Aleyrodidae) and inoculation efficiency of tomato chlorosis virus. Insects. 2020. № 11(9). Vol. 559. P. 2-14. DOI:10.3390/insects11090559

19. Gilbertson R.L., Rojas M., Natwick, E. Development of integrated pest management (IPM) strategies for whitefly (Bemisia tabaci)-transmissible geminiviruses. In the whitefly, Bemisia tabaci (Homoptera: Aleyrodidae) interaction with geminivirus-infected host plants. In: W.M.O. Thompson, ed. Springer: Dordrecht, The Netherlands, 2011. P. 323-356. DOI: 10.1007/978-94007-1524-0 12.

20. Godfrey L.D. et al. UC IPM pest management guidelines: cotton: insects and mites. UC ANR Publ. 3444, 2001. 70 p.

21. Summers C.G. Integrated pest management in forage alfalfa. Int. Pest Man. Rev. 1998. Vol. 3. P. 127-154. DOI: 10.1023/A:1009654901994.

22. Вигера С.М., Рубан М.Б. Насіннєва люцерна. Агробіологічна система захисту від 
шкідливих організмів. Захист рослин. 1997. № 5. С. 24-25.

23. Голобородько С.П., Снеговой В.С., Сахно Г.В. Люцерна. Херсон: Айлант, 2007. 328 с.

24. Вигера С.М. Захист посівів насіннєвої люцерни в умовах біологічного та інтенсивного землеробства. Захист рослин. 2002. № 2. С. 6-8.

25. Девяткин А.М., Маркова И.А., Белый А.И. Вредители, болезни и сорняки люцернового агроценоза. Краснодар, 2013. 477 с.

26. Артохин К.С. Метод кошения энтомологическим сачком. Защита и карантин растений. 2010. Вып. 11. С. 45-48.

27. Трибель С.О. та ін. Методики випробування і застосування пестицидів. За ред. проф. С.О. Трибеля. К.: Світ, 2001. 448 с.

28. Алехин В.Т., Михайликова В.В., Михина Н.Г. Экономические пороги вредоносности вредителей, болезней и сорных растений в посевах сельскохозяйственных культур: справочник. М.: ФГБНУ «Росинформагротех», 2016. 76 с.

\section{References}

1. Katungi E et al. Common bean in Eastern and Southern Africa: a situation and outlook analysis. International Centre for Tropical Agriculture, 2009; $61 \mathrm{p}$.

2. Ochilo WN, Nyamasyo GH. Pest status of bean stem maggot (Ophiomyia spp.) and black bean aphid (Aphis fabae) in Taita district, Kenya. Tropical and Subtropical Agro Ecosystems. 2011; 13(1): 91-97.

3. Munyasa AJ. Evaluation of drought tolerance mechanisms in mesoamerican dry bean genotypes. University of Nairobi, Nairobi, 2013. 191 p.

4. Spiridonov YuY. et al. Technology of cultivation of spring durum wheat with use of drugs Secator turbo, Baritone, Falcon, Nagro and others. Agrarnyy nauchnyy zhurnal. 2017; 3: 30-36. DOI: 10.28983 /asj.v0i3.47.

5. Strizhkov NI et al. Integrated technology for protection of field crops from diseases, pests and weeds based on biological and chemical methods. Saratov, 2017; 56 p.

6. Spiridonov YuYa et al. Development of integrated technology for protection of field crops from diseases, pests and weeds based on biological and chemical methods. Agrarnyiy nauchnyiy zhurnal. 2017; 9: 37-42.

7. Dudkin IV et al. Ecological aspects of formation of systems of agriculture and plant protection. Vestnik Kurskoy gosudarstvennoy selskohozyaystvennoy akademii. 2017; 7: 2-7.

8. Peter KH, Swella GB, Mushobozy DM. Effect of plant populations on the incidence of bean stem maggot (Ophiomyia spp.) in common bean intercropped with maize. Plant Protection Science. 2009; 45(4): 148-155. DOI: 10.17221/19/2009-PPS.

9. Dhungana SK et al. Comparative effect of different insecticides on the growth and yield of soybeans. Plant Protect. Sci. 2020; 56: 206-213. DOI: 10.17221/77/2019-PPS.

10. Hudenko MN et al. Productivity of milk thistle depending on the methods of soil treatment and chemical protection in the dry steppe of the Volga region. Agrarnyiy nauchnyiy zhurnal. 2016; 12: 43-49.

11. Spiridonov YuYa et al. Features of the influence of chemical plant protection products on the dynamics of nutrients in plants, their chemical composition and development conditions. Agrarnyiy nauchnyiy zhurnal. 2018. 10. 37-40. DOI: 10.28983/asj.v0i10.606.

12. Abate T, Ampofo JKO. Insect pests of beans in Africa: their ecology and management. Annual Review of Entomology. 1996; 41: 45-73. DOI: 10.1146/annurev.en.41.010196.000401.

13. Acreman TM, Dixon AF. Developmental patterns in the wheat and resistant to cereal aphids. Crop Protect. 1985; 4: 322-328. DOI: 10.1016/0261-2194(85)90034-1.

14. Aheer GM et al. Effects of swoing dates on aphids and grain yield in wheat. Journal of Agricultural Research. 1993; 31: 75-79.

15. Nderitu JH, Kayumbo HY, Mueke JM. Beanfly infestation on common beans Phaseolus vulgaris in Kenya. Insect Science and Its Application. 1990; 11: 35-41. DOI: $10.1017 /$ S1742758400019810. 
16. Forbes VE et al. Ecological models in support of regulatory risk assessments of pesticides: developing a strategy for the future. Integrated Environmental Assessment and Management. 2009; 5: 167-172. DOI: 10.1897/ieam 2008-029.1.

17. Stoddard FL et al. Integrated pest management in faba bean. Field Crops Research. 2010; 115 : 308-318. DOI: 10.1016/j.fcr.2009.07.002.

18. Maluta NKP et al. Foliar spraying of tomato plants with systemic insecticides: effects on feeding behavior, mortality and oviposition of Bemisia tabaci (Hemiptera: Aleyrodidae) and inoculation efficiency of tomato chlorosis virus. Insects. 2020: 11(9),559; 2-14. DOI: 10.3390/insects11090559

19. Gilbertson RL, Rojas M, Natwick E. Development of integrated pest management (IPM) strategies for whitefly (Bemisia tabaci)-transmissible geminiviruses. In The Whitefly, Bemisia tabaci (Homoptera: Aleyrodidae) Interaction with Geminivirus-Infected Host Plants. IN: WMO Thompson, ed. Springer: Dordrecht, The Netherlands, 2011; 323-356. DOI: 10.1007/978-94007-1524-0_12.

20. Godfrey LD et al. UC IPM pest management guidelines: cotton: insects and mites. UC ANR Publ. 3444, 2001. 70 p.

21. Summers CG. Integrated pest management in forage alfalfa. Int. Pest Man. Rev. 1998; 3: 127 154. DOI: $10.1023 / \mathrm{A}: 1009654901994$.

22. Vigera SM, Ruban MB. Seed alfalfa. Agrobiological system of protection against pests. Zakhyst roslyn. 1997; 5: 24-25.

23. Goloborodko SP, Snegovoy VS, Sahno GV. Alfalfa. KHerson: Aylant, 2007. 328 P.

24. Vyhera SM. Protection of alfalfa seed crops in the conditions of biological and intensive agriculture. Zakhyst roslyn. 2002; 2: 6-8.

25. Devyatkin AM, Markova IA, Belyiy AI. Pests, diseases and weeds of alfalfa agrocenosis. Krasnodar, 2013. 477 p.

26. Artohin KS. The method of mowing with an entomological net. Zaschita i karantin rasteniy. 2010; 11: 45-48.

27. Tribel SO et al. Methods of testing and application of pesticides. In: SO Tribel, ed. Kyiv: SvIt, 2001. $448 \mathrm{p}$.

28. Alekhin VT, Mikhaylikova VV, Mihina NG. Economic thresholds of pests, diseases and weeds in crops: a guide Moscow: FGBNU «Rosinformagroteh», 2016. 76 p.

\section{ЗАХИСТ НАСІННЕВОЇ ЛЮЦЕРНИ ДРУГОГО РОКУ ЖИТТЯ ВІД ШКІДНИКІВ}

Тищенко А.В. ${ }^{1}$, Тищенко О.Д. ${ }^{1}$, Куц Г.М. ${ }^{1}$, Пілярська О.О. ${ }^{1}$, Гальченко Н.М. ${ }^{2}$

${ }^{1}$ Інститут зрошуваного землеробства НААН, Україна

${ }^{2}$ Асканійська Державна сільськогосподарська дослідна станція Інституту зрошуваного землеробства НААН, Україна

Метою дослідження було виявити ефективність різних інсектицидів проти шкідників на насінницьких посівах люцерни другого року життя травостою.

Матеріали і методи. Дослідження проводили у 2017-2019 pp. на дослідному полі Інституту зрошуваного землеробства НААН. Метод закладки польового досліду - розщеплені ділянки. Головні ділянки (фактор А) - умови зволоження (без зрошення і зрошення); субділянки (фактор В й С) - застосування інсектициду. Посів широкорядний 3 міжряддям 70 см. Площа посівної ділянки - $60 \mathrm{~m}^{2}$, облікової - $50 \mathrm{~m}^{2}$, повторення триразове. Сорт люцерни Елегія. Травостій другого року життя (весняний посів), насіння отримане 3 першого укосу.

Видовий склад шкідливих комах виявляли за проведення обстежень, чисельність - за допомогою ентомологічного сачка (10 помахів). Обробку інсектицидами проводили: 
першу - у фазу початок бутонізації, другу - до початку цвітіння навісним обприскувачем ОН-600 з витратою робочої рідини 250 л/га.

Обговорення результатів. Найвищу врожайність насіння отримали на варіанті при першій обробці інсектицидним препаратом 3 діючими речовинами Хлорпірифос, 500 г/л й Циперметрин, 50 г/л та нормою витрати 1,00 л/га та при другій обробці препаратами 3 діючими речовинами Хлорантраніліпрол, 200 г/л й Лямбда-цигалотрин, 50 г/л нормою 0,17 й 0,15 л/га, відповідно. При зрошенні на цьому варіанті утворилося 8,8 бобів/китицю $і$ 4,64 насіння/біб, а насіннєва продуктивність склала 635,16 кг/га, що було вище контрольного варіанту на 210,56 кг/га. В умовах природного зволоження у рослин утворилося 7,4 бобів/китицю і 2,98 насіння/біб. При цьому врожайність насіння становила 452,48 кг/га, та була вище контролю на 132,49 кг/га.

Висновки. Найефективнішим у боротьбі зі шкідниками виявився препарат 3 діючими речовинами Хлорпірифос, 500 г/л й Циперметрин, 50 г/л та нормою витрати 1,00 л/га. Але наявність у даного препарату фумігаційної дії негативно позначилась на чисельності комах-запилювачів, що в подальшому вплинуло на продуктивність рослин, тому цей препарат не слід застосовувати перед цвітінням.

Ключові слова: лючерна, насіння, інсектициди, зрочення, природнє зволоження

\section{ЗАЩИТА СЕМЕННОЙ ЛЮЦЕРНЫ ВТОРОГО ГОДА ЖИЗНИ ОТ ВРЕДИТЕЛЕЙ}

Тищенко А.В. ${ }^{1}$, Тищенко Е.Д. ${ }^{1}$, Куц Г.М. ${ }^{1}$, Пилярская Е.А. ${ }^{1}$, Гальченко Н.Н. ${ }^{2}$

${ }^{1}$ Институт орошаемого земледелия НААН, Украина

2 Асканийская Государственная сельскохозяйственная опытная станция Институт орошаемого земледелия НААН, Украина

Целью исследования было выявить эффективность различных инсектицидов против вредителей на семенных посевах люцерны второго года жизни травостоя.

Материалы и методы. Исследования проводили в 2017-2019 гг. на опытном поле Института орошаемого земледелия НААН. Метод закладки полевого опыта расщепленные делянки. Главные делянки (фактор А) - условия увлажнения (без орошения и орошение); суб-делянки (фактор В и С) - применение инсектицида. Посев широкорядный с междурядием 70 см. Площадь делянки - $60 \mathrm{~m}^{2}$, учетной $-50 \mathrm{~m}^{2}$, повторение трехкратное. Сорт люцерны Елегія. Травостой второго года жизни (весенний посеыв), семена получены с первого укоса.

Видовой состав вредных насекомых определяли при проведении обследований, численность - при помощи энтомологического сачка (10 взмахов). Обработку инсектицидами проводили: первую - в фазе начала бутонизации, вторую - до начала цветения навесным опрыскивателем ОН-600 с расходом рабочей жидкости 250 л/га.

Обсуждение результатов. Наивысшшую урожайность семян получили на варианте при первой обработке инсектицидным препаратом с действующими веществами хлорпирифос, 500 г/л и циперметрин, 50 г/л и нормой расхода 1,00 л/га и при второй обработке препаратами с действующими веществами Хлорантранилипрол, 200 г/л и Лямбдацигалотрин, 50 г/л нормой 0,17 и 0,15 л/га соответственно. При орошении на этом варианте образовалось 8,8 бобов/кисть и 4,64 семян/боб, а семенная продуктивность составила 635,16 кг/га. Это превышало контрольный вариант на 210,56 кг/га. В условиях естественного увлажнения у растений образовалось 7,4 бобов/кисть и 2,98 семян/боб. При этом урожайность семян составила 452,48 кг/га, что выше контроля на 132,49 кг/га.

Выводы. Наиболее эффективным в борьбе с вредителями оказался препарат с действующими веществами Хлорпирифос, 500 г/л и Циперметрин, 50 г/л и нормой расхода 1,00 л/га. Но наличие у данного препарата фуммигативного действия 
отрицательно сказалось на численности насекомых-опылителей, что в дальнейшем повлияло на продуктивность растений.

Ключевые слова: лючерна, семена, инсектициды, орочения, естественное увлажнение

\section{ANTI-PEST PROTECTION OF TWO-YEAR OLD ALFALFA GROWN FOR SEEDS}

Tyshchenko A.V. ${ }^{1}$, Tyshchenko O.D. ${ }^{1}$, Kuts G.M. ${ }^{1}$, Piliarska O.O. ${ }^{1}$, Galchenko N.M. ${ }^{2}$

${ }^{1}$ Institute of Irrigated Agriculture of NAAS, Ukraine

${ }^{2}$ Askania State Agricultural Experimental Station of the Institute of Irrigated Agriculture of NAAS, Ukraine

Purpose. To evaluate the effectiveness of different insecticides against pests on two-year old alfalfa grown for seeds.

Materials and methods. The study was conducted in the experimental field of the Institute of Irrigated Agriculture of NAAS in 2017-2019. The filed experiments were laid out in split plots. The main plots (factor A) were for water supply conditions (no irrigation vs. irrigation); subplots (factors B and C) were for application of insecticides. Seeds were sown in wide rows with interrows of $70 \mathrm{~cm}$.

Results and discussion. When the two-year old alfalfa canopy (budding onset) was inspected before insecticide treatment, the average numbers of pests were as follows: alfalfa plant bug 3.0 specimens $/ 10$ sweeps, alfalfa aphid - 20.0 specimens $/ 10$ sweeps, beet webworm - 3.0 specimens/10 sweeps, alfalfa weevil (imagoes/grubs) - 1.0/3.0 specimens/10 sweeps, and alfalfa seed weevil - 1.0 specimens/10 sweeps. Treatment 1 reduced the pest numbers in the canopy: alfalfa plant bug - by 70.0-93.3\%, alfalfa aphid - by $93.0-97.5 \%$, beet webworm - by 80.0 96.7, alfalfa weevil (imagoes/grubs) - by $60.0-90.0 \% / 73.3-93.3 \%$, and alfalfa seed weevil - by $76.0-94.0 \%$, depending on the insecticide. Treatment 2 helped to reduce the pest numbers in the canopy. Insecticide containing active substances chlorpyrifos $500 \mathrm{~g} / \mathrm{L}$ and cypermethrin $50 \mathrm{~g} / \mathrm{L}$ applied at a dose of $1.00 \mathrm{~L} / \mathrm{ha}$ was the most effective. The highest seed yield was achieved after treatment 1 with insecticide containing active ingredients chlorpyrifos $500 \mathrm{~g} / \mathrm{L}$ and cypermethrin $50 \mathrm{~g} / \mathrm{L}$ applied at a dose of $1.00 \mathrm{~L} / \mathrm{ha}$ and treatment 2 with insecticide containing active ingredients chlorantraniliprole $200 \mathrm{~g} / \mathrm{L}$ and lambda-cyhalothrin $50 \mathrm{~g} / \mathrm{L}$ applied at a dose of 0.17 $\mathrm{L} / \mathrm{ha}$ and $0.15 \mathrm{~L} / \mathrm{ha}$, respectively.

Conclusions. The highest seed yield was achieved after treatment 1 with insecticide containing active ingredients chlorpyrifos $500 \mathrm{~g} / \mathrm{L}$ and cypermethrin $50 \mathrm{~g} / \mathrm{L}$ applied at a dose of $1.00 \mathrm{~L} / \mathrm{ha}$ and treatment 2 with insecticide containing active ingredients chlorantraniliprole $200 \mathrm{~g} / \mathrm{L}$ and lambda-cyhalothrin $50 \mathrm{~g} / \mathrm{L}$ applied at a dose of $0.17 \mathrm{~L} / \mathrm{ha}$ and $0.15 \mathrm{~L} / \mathrm{ha}$, respectively.

Key words: alfalfa, seeds, insecticides, irrigation, rainfed conditions. 